\title{
Review of: "Plasmodiophora brassicae in Mexico, from anecdote to fact"
}

\author{
Nazanin Zamani-Noor
}

Potential competing interests: The author(s) declared that no potential competing interests exist.

Manuscript ID: bioRxiv preprint doi: https://doi.org/10.1101/2021.11.29.469274

Title: Plasmodiophora brassicae in Mexico, from anecdote to fact

In the current study, the authors evaluated the presence of clubroot disease and its causal agent, Plasmodiophora brassicae, in several Brassica crops in different regions of Mexico.

Surely, this manuscript will be a useful addition to the literature on this subject. However, in my opinion the authors should revise deeply the manuscript based on the reviewer's comments/suggestions and resubmit the paper.

\section{Comments:}

1. First of all the English language overall need to be improved and should check by a professional proofreader. Please avoid informal language at the scientific paper (e.g., we did detective works).

1. As following you will find some publications, which help you to improve strongly your manuscript:

- Strehlow B, de Mol F, Struck C, 2013. History of oilseed rape cropping and geographic origin influence the genetic structure of Plasmodiophora brassicae populations. Phytopathology 104, 532-8.

- Ričařová, V., Kaczmarek, J., Strelkov, S. E., Kazda, J., Lueders, W., Rysanek, P., Manolii, V., \& Jedryczka, M. (2016). Pathotypes of Plasmodiophora brassicae causing damage to oilseed rape in the Czech Republic and Poland. European Journal of Plant Pathology, 145, 559-572.

- Zamani-Noor N, 2017. Variation in pathotypes and virulence of Plasmodiophora brassicae populations in Germany. Plant Pathology 66, 316-24.

- Zamani-Noor, N.; Rodemann, B. Reducing the build-up of Plasmodiophora brassicae inoculum by early management of oilseed rape volunteers. Plant Pathol. 2018, 67, 426-432.

1. The 'Introduction' section should be improved. The Introduction should answer the question 'Why:' why you choose that topic for research; why it is important; why you adopted a particular method or approach; and so on. You can also think of the Introduction as the section that points out the gap in 
knowledge that the rest of the paper will fill, or the section in which you define and claim your territory within the broad area of research.

1. Introduction, Line 75-79: Instead of explaining the results in this part, you should write clearly the objectives of your project.

1. Materials and Methods, line 110: How did you disinfect the seeds? Did you use any disinfection detergent or special solution?

1. Materials and Methods, line 115: How about irrigation? How often did you irrigate the pots?

1. Materials and Methods, line 118: How did you assess the survival rate of each plant cultivar?

2. Materials and Methods, line 124: Which cultivar of cabbage did you use to fulfil the Koch postulate? How many plants did you use as replicate?

1. Materials and Methods, line 118: How did you inoculate the soil? When did you sown the cabbage seeds into the infested soil?

2. Materials and Methods: In this part, it is not clear how you did assess the galls? Which scale did you use? Why you did not calculate the disease severity index or disease incidence for each plant cultivar?

1. Results, line 147 to 149 , the meaning of the sentence is not clear!

1. Results, line 163-164: What do mean with 'development was seriously affected' how did you assess the plant development?

1. Results, line 169-171: How did you know that the plants die because of $P$. brassicae?

1. Results, line 177: Here you mentioned that 'the DNA extracted from cauliflower grown in SPC6 soil

1. Results, line 187-189: You should explain in 'Discussion' why 'only symptomatic cauliflower plants grown in the soil collected from SPC4 were positive for the presence of $P$. brassicae by PCR, while symptomatic cabbage plants were negative'.

1. The description of figures/tables are very poor. Figure/Table legends should support your figure entirely, meaning that the reader of your thesis should be able to understand your figure, paired with its legend, without going to the results or methods sections to see what you say about your observations or how the experiment was done. 
As following, you will find the link, which help you to write a proper legend:

http://www.biosciencewriters.com/Tips-for-Writing-Outstanding-Scientific-Figure-Legends.aspx

1. Finally yet importantly, the 'Discussion' section should be improved. The discussion is a review of your findings, and it should show you really understand them. Explain how your findings/results relate to what is already known in the field as well as to what you expected to find. You should refer back to your introduction and establish if what you found was consistent with the existing literature, or if it was somewhat unexpected or controversial. If your findings were unexpected and/or contradictory, you need to explain why you think that was. 